\title{
A Noninvasive Cardiac Output Trend Monitor Targeting Telemedicine Applications
}

\author{
Ovidio López ${ }^{1}$, Rafael Maestre ${ }^{1}$, Andrés L Bleda ${ }^{1}$, Ricardo Ruiz ${ }^{2}$, Javier Corral ${ }^{2}$ \\ ${ }^{1}$ Technological Centre of Furniture and Wood of the Region of Murcia, Yecla (Murcia), Spain \\ ${ }^{2}$ RGB Medical Devices SA, Madrid, Spain
}

\begin{abstract}
This study aimed to investigate and validate a noninvasive affordable cardiac output $(\mathrm{CO})$ trend monitor intended for telemedicine applications. The approach of this work will widely increase the availability of $\mathrm{CO}$ measurements, currently only available through expensive hospital equipment. The estimation method of the CO trend is based on the transient analysis of a PPG (photoplethysmography) signal during venous occlusion. The PPG signal is acquired with an LED and a photodiode as in typical pulse oximeters, whereas a pneumatic cuff and pressure pump implement the occlusion and release cycles. The $\mathrm{CO}$ trend is given by the relative comparison of different CO measurements of the same individual. All the components used in this work have been already integrated into a portable device with wireless communications so it can be suitable for telemedicine applications. Different measures were taken on different individuals at different times of the day, several days per week during some weeks. The CO trend consistently reflected the expected daily $C O$ variation patterns and events such as food intake and mild physical activities. The proposed methodology can be used to determine sudden CO changes or to analyze the underlying overall CO trend with measurements taken over multiple days.
\end{abstract}

\section{Introduction}

According to the World Health Organization (WHO), cardiovascular diseases are the first cause of reported mortality worldwide [1]. In particular, heart failure (HF) is a major public health problem. Projections show that the prevalence of HF will increase 46\% from 2012 to 2030, exceeding a total of 8 million adults worldwide with $\mathrm{HF}$ by 2030 . Additionally, the total percentage of the population with $\mathrm{HF}$ is predicted to increase from $2.42 \%$ in 2012 to $2.97 \%$ in 2030 .

During the last decades there has been a growing number of telemedicine applications users, which are changing the way people seek and get medical advice, diagnoses, and prescriptions. Cardiac output (CO) is the blood flow that the heart ejects per minute and is directly related to the heart rate (HR) and stroke volume (SV) by the following expression $\mathrm{CO}=\mathrm{HR} \times \mathrm{SV}$. Continuous $\mathrm{CO}$ monitoring is especially useful for chronic heart-related diseases. However, high quality medical-grade equipment is costly or invasive which is not suitable for telemedicine applications. Current telemedicine devices can provide variables to estimate the $\mathrm{CO}$ trend. $\mathrm{CO}$ trend is one of the best techniques to identify cardiovascular health changes which serves for better diagnostics.

The primary objective of this work is to present a preliminary investigation of a $\mathrm{CO}$ trend measurement technique that uses very common, simple and affordable devices including a pneumatic cuff and a pulse oximeter. This technique obtains the plethysmography readings from a pulse oximeter to estimate the amount of blood in a fingertip during cycles of forearm venous occlusions and releases. The speed of blood accumulation in the finger is affected by the actual $\mathrm{CO}$, even though other secondary factors may also influence.

\section{CO trend estimation method}

The CO trend is based on the transient analysis of a PPG (photoplethysmography) signal during venous occlusion. Venous occlusion is achieved for pressures in the range $40 \mathrm{mmHg}$ to $60 \mathrm{mmHg}$ with a pneumatic cuff without distorting the blood flow of the arteries, see for example [2,3]. If the cuff is inflated too slowly, venous occlusion will not be effectively achieved and kept during the accumulation of blood in the forearm. This only happens when inflation time is faster than 6 seconds, and results are independent of inflation time so long as it remains below that time threshold (see [4]). That condition is met in our experiments.

A pulse oximeter measures the relative amount of transmitted light through the fingertip. It contains a pair of small LEDs located in a finger probe, which emit red and near-infrared (near-IR) light [5]. The transmitted light through the finger is then detected by a photodiode. When 


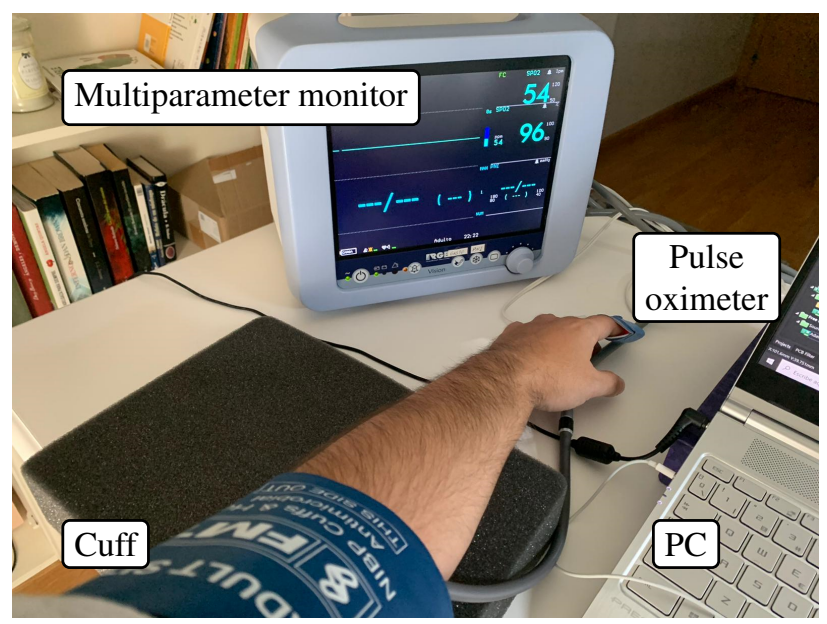

Figure 1: Test experimental setup. A test involves a user, a multiparameter vital signs monitor (multiparameter monitor), a personal computer (PC), a pneumatic cuff (cuff), and a pulse oximeter.

the volume of vascular bed increases, a lower signal is observed because more light is absorbed. We select the signal corresponding to the near-IR light because it is less affected by oxygen concentration in the blood, which will be reduced during venous occlusion. This signal is the PPG signal. We assume that the relative changes in the PPG signal are proportional to the relative changes in volume of the fingertip vascular bed. Thus, the relative rate of change (slope, S) of the PPG signal is proportional to the relative rate of change of the volume of the fingertip vascular bed. In other words, $S$ is proportional to the local blood flow, which in turn, we assume to be proportional to the $\mathrm{CO}$ of the heart. Situations where this assumption is not true include thermoregulation in cold ambients or other situations that produce vasoconstriction $[6,7]$. Other parameters like the area between the actual PPG curve and its baseline value (i.e. PPG average during no venous occlusion), or the PPG amplitude (i.e. difference between PPG baseline and its asymptotic value) can serve for $\mathrm{CO}$ trend estimation. In this work, we present the results on the $\mathrm{S}$ parameter, since similar results can be achieved with the other two. For the same individual under similar measurement conditions, mainly including time of the day, temperature, food and caffeine intake, the $\mathrm{S}$ parameter trend should be a good estimation of the real $\mathrm{CO}$ trend.

The $\mathrm{CO}$ trend can be observed by relative comparison of different values of the $S$ parameter for the same individual. Usually, the $S$ parameter is negative, therefore we give its absolute value. Thus, an increase in $\mathrm{CO}$ should be reflected in an increase of the $\mathrm{S}$ parameter.

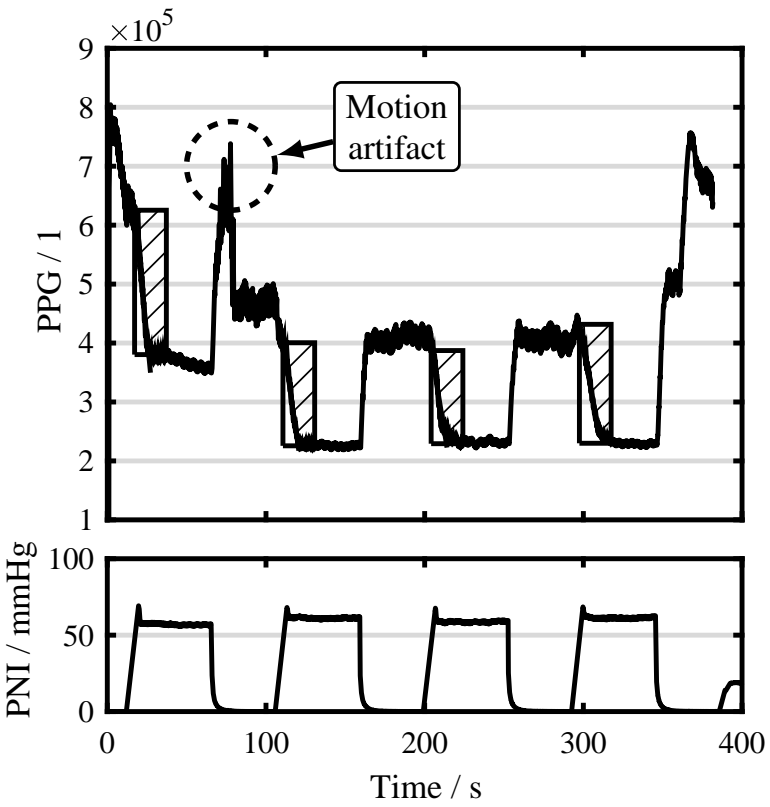

Figure 2: Typical PPG and PNI signals of a user test. The four venous occlusion periods are shown. Movement artifacs can lead to low-quality PPG signals for the CO trend estimation.

\section{Experimental setup}

We use a modified multiparameter vital signs monitor [8] to perform venous occlusion, like shown in fig. 1 . The user sits in an upright position with the arm placed at the height of the heart. The user is asked not to move or talk. The user has a pneumatic cuff in the left forearm and a pulse oximeter in his or her left index finger. The arm rests over a cushion. Each period of venous occlusion has a typical duration of $50 \mathrm{~s}$ [3]. Although the tests for investigation and validation of this $\mathrm{CO}$ trend measurement methodology have been done with the modified multiparameter monitor, this type of methodology and data acquisition have also been tested in a portable device with wireless communications similar to [9], so the presented technique can be suitable for telemedicine applications.

In each $\mathrm{CO}$ trend measurement, venous occlusion is repeated four times, like shown in fig. 2. In the figure, PNI is the pressure of the pneumatic cuff. For each measurement, the $\mathrm{S}$ value is the mean of the $\mathrm{S}$ values with the $95 \%$ confidence interval for small sample size.

\section{Results}

The $S$ results obtained from three healthy volunteers between 27-40 years-old are shown in figs. 3 to 5. They followed an approximately fixed schedule monitoring routine during different working days. Interestingly, the values of 


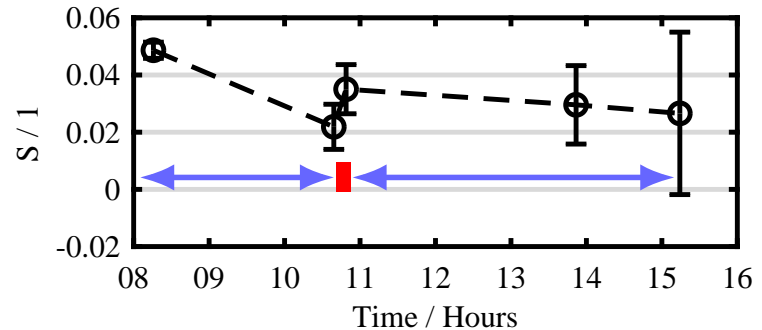

(a) Day 1

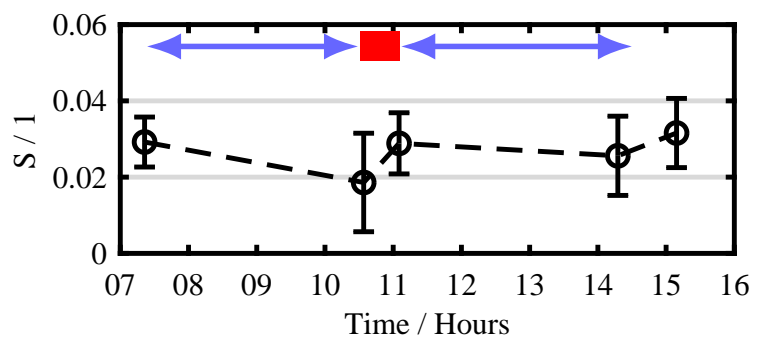

(c) Day 3

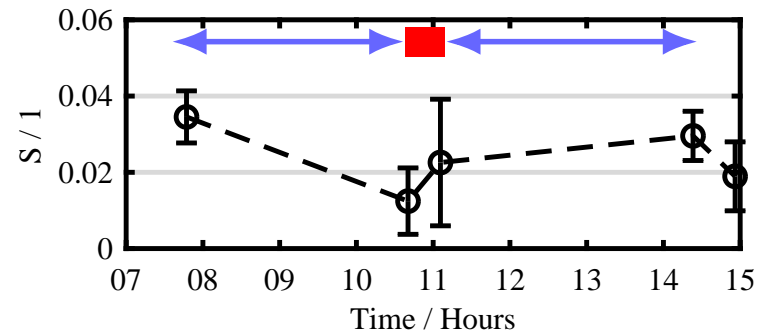

(b) Day 2

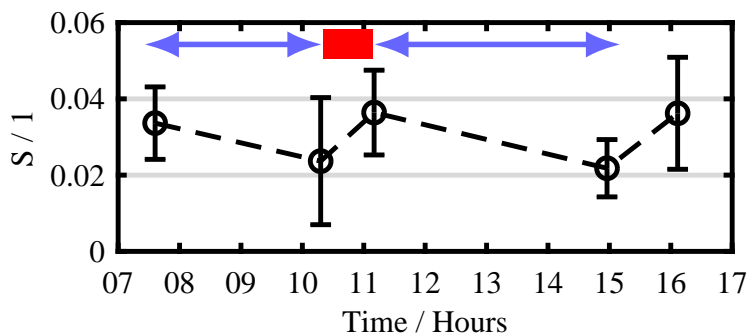

(d) Day 4

Figure 3: Parameter S for user 1 for 4 days. In each day, the tests were took during the morning and noon. Legend: Meal or coffee; $\longrightarrow$ : Sitting.

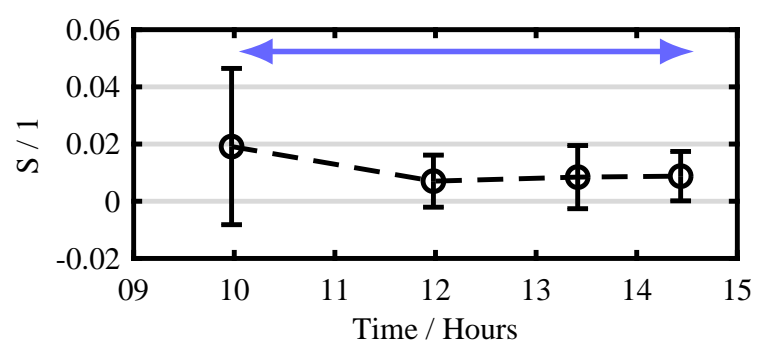

(a) Day 1

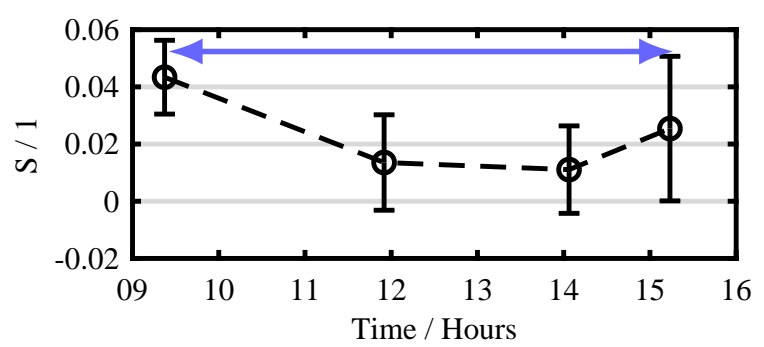

(b) Day 2

Figure 4: Parameter S for user 2 for 2 days. Legend: $\square:$ Meal or coffee; $\longrightarrow$ : Sitting.

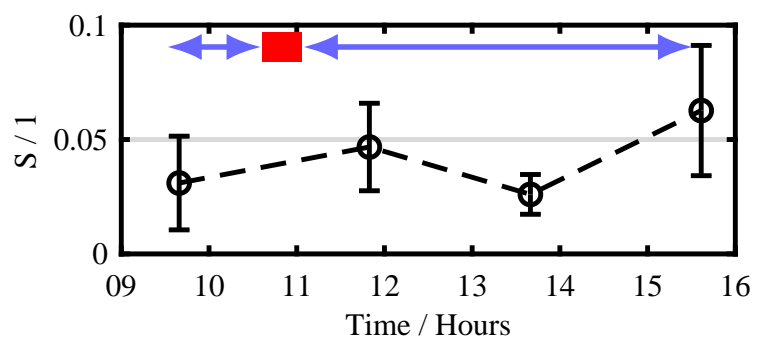

(a) Day 1

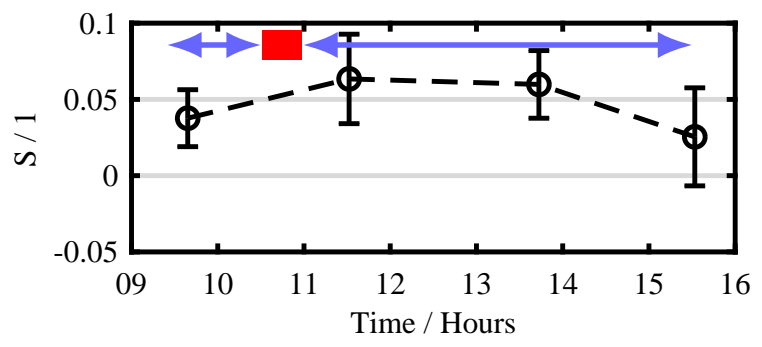

(b) Day 2

Figure 5: Parameter $\mathrm{S}$ for user 3 for 2 days. Legend: $\square$ : Meal or coffee; $\longrightarrow$ : Sitting. 
the $S$ parameter are usually between $0.02-0.06$, which reflects a consistent $\mathrm{CO}$ state across different days for the same user.

User 1 data, in fig. 3 shows two measurements taken around $11 \mathrm{am}$, right before and after a quick meal. A clear increase of the parameter $\mathrm{S}$ is observed in relation to the increase in blood flow as a consequence of digestion and movement. A similar increase is also observed in user 3 data, even though the measurements were taken more than two hours apart, approximately at $10 \mathrm{am}$ and $12 \mathrm{am}$, this last one right after lunch. User 2 did not have any food between the first two measurement, and this is why his data show a decrease, instead of an increase of S. For user 1, the hours from breakfast to the working lunch show a consistent decrease in $\mathrm{CO}$, which is also observed in user 2. In user 3, it is likely that this decrease could be observed if the user would have done a measure just before the working lunch. Similar values are observed for user 2 and 3. In summary, for all users an increase of $\mathrm{CO}$ is observed after food intake, whereas a decrease is shown when sitting for several hours.

\section{Conclusions}

The presented CO trend estimation methodology shows consistent results when compared across multiple experiments under similar measurement conditions. For every individual that participated in the experiments, an increase of $\mathrm{CO}$ is observed after food intakes. On the other hand, the $\mathrm{CO}$ is estimated to decrease after a long sitting period. This methodology is consistent with the expected qualitative variations of the $\mathrm{CO}$ trend, which is very promising to be applied in telemedicine applications. A patent application regarding the presented system and method has been filled.

Actual results can only be used to estimate the $\mathrm{CO}$ trend for a given individual, that is whether there has been an increase or decrease of $\mathrm{CO}$, and how significant this has been. Unfortunately, absolute $\mathrm{CO}$ estimates cannot be achieved with the presented technique, and results cannot be used to compare the $\mathrm{CO}$ state of two different individuals. It is worth mentioning that measurements can be affected by different conditions in limbs, skin temperature, stress level, medication, etc. Other factors are related with how users carry out the measurement such as user postures or movement, variations of pulse oximeter or cuff positioning or bad usage, etc.

Further research is still needed for validating the presented methodology, as well as to achieve quantitative $\mathrm{CO}$ results. A more rigorous study is being planned to carry out a big set of experiments with individuals that have different medical conditions, where this technique is compared against absolute $\mathrm{CO}$ and trend $\mathrm{CO}$ values obtained from medical-grade equipment.

\section{Acknowledgments}

We thank the staff of RGB Medical Devices for their role in the collaborative development of the "EneHeart" project, an R\&D project supported by the "Ministerio de Economia y Competitividad" from the Spanish Government under grant RTC-2016-5439-1.

\section{References}

[1] Virani SS, Alonso A, Benjamin EJ, Bittencourt MS, Callaway CW, Carson AP, Chamberlain AM, Chang AR, Cheng $\mathrm{S}$, Delling FN, et al. Heart disease and stroke statistics-2020 update: a report from the american heart association. Circulation 2020;E139-E596.

[2] Wilkinson IB, Webb DJ. Venous occlusion plethysmography in cardiovascular research: methodology and clinical applications. British journal of clinical pharmacology 2001; 52(6):631.

[3] Van Vo T, Hammer PE, Hoimes ML, Nadgir S, Fantini S. Mathematical model for the hemodynamic response to venous occlusion measured with near-infrared spectroscopy in the human forearm. IEEE transactions on biomedical engineering 2007;54(4):573-584.

[4] Casavola C, Paunescu LA, Fantini S, Gratton E. Blood flow and oxygen consumption with near-infrared spectroscopy and venous occlusion: spatial maps and the effect of time and pressure of inflation. Journal of biomedical optics 2000; 5(3):269-276.

[5] Chan ED, Chan MM, Chan MM. Pulse oximetry: Understanding its basic principles facilitates appreciation of its limitations. Respiratory Medicine 2013;107(6):789 - 799. ISSN 0954-6111. URL http://www.sciencedirect.com/science/ article/pii/s095461111300053X.

[6] Charkoudian N. Skin blood flow in adult human thermoregulation: how it works, when it does not, and why. In Mayo clinic proceedings, volume 78. Elsevier, 2003; 603-612.

[7] Romanovsky AA. Skin temperature: its role in thermoregulation. Acta physiologica 2014;210(3):498-507.

[8] RGB Medical Devices. Monitor multiparamétrico ECG VISION TOF. https://www. medicalexpo.es/prod/rgb-medical-devices/ product-69843-872940.html. Accessed: 2020-0905.

[9] Bleda AL, Maestre R, Corral J, Ruiz R. A quality and ergonomic heart monitoring device with user-friendly app for telemedicine. In Multidisciplinary Digital Publishing Institute Proceedings, volume 31. 2019; 67.

Address for correspondence:

Ovidio López

C/ Perales, S.N., 30510, Yecla (Murcia), Spain

o.lopez@cetem.es 